\title{
Assessment and \\ management: Primary \\ hypothyroidism in women
}

\section{By Cynthia S. Watson, DNP, FNP-BC, and Janis Guilbeau, DNP, FNP-BC}

Hypothyroidism is a common endocrine disorder affecting nearly 2 in 100 women. Primary hypothyroidism can be diagnosed and effectively managed by primary care providers. Pharmacologic treatment is essential and lifelong, with most patients achieving euthyroid state and enjoying good quality of life.

KEY WORDS: primary hypothyroidism; Hashimoto thyroiditis, thyroidstimulating hormone, euthyroid, levothyroxine

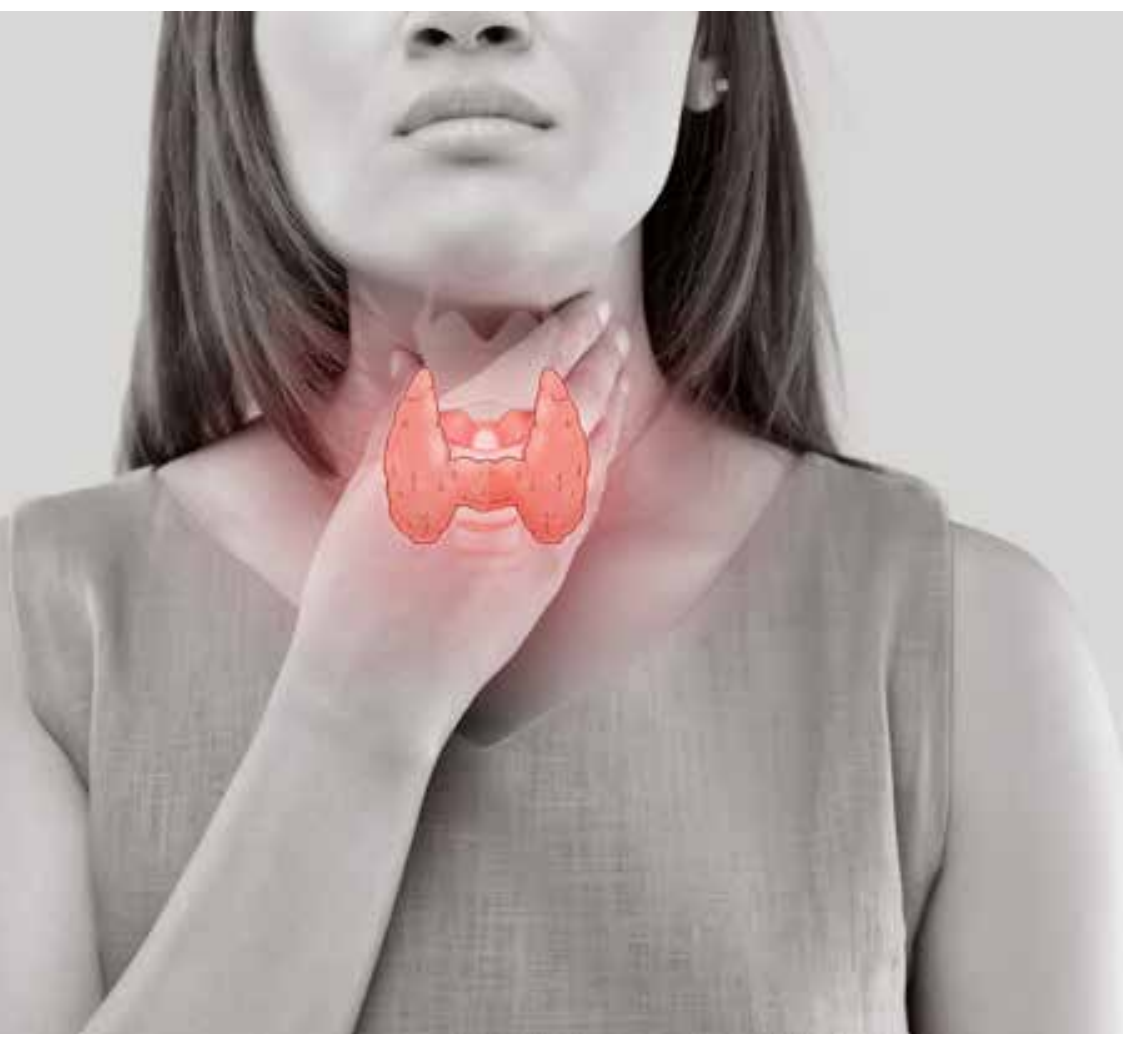

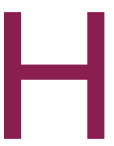

ypothyroidism affects significantly more women than men with a prevalence rate of approximately 2 in $100 .{ }^{1}$ It occurs more commonly in postpartum women, the elderly, and those with autoimmune disorders. ${ }^{2}$ Thus, nurse practitioners (NPs) who provide care for women throughout the adult lifespan will encounter patients with hypothyroidism. The woman may already have a confirmed diagnosis of hypothyroidism and be on medication or may have signs and symptoms that warrant an investigation for possible hypothyroidism. Having knowledge about thyroid physiology, clinical signs and symptoms of hypothyroidism, and laboratory testing enhances the NP's ability to diagnose and treat this condition effectively. The treatment usually involves life-long medication with regular thyroid function test monitoring. The purpose of this article is to review this information for use in the primary care setting. The focus is on nonpregnant women.

The thyroid is a complex endocrine gland. Thyroid hormone has a major role in regulating metabolism affecting many body systems. The hypothalamic-pituitary-thyroid feedback system regulates the secretion of thyroid hormone. A 
Table. Thyroid function tests for hypothyroidism

\begin{tabular}{l|l|l|l} 
Type of hypothyroidism & \multicolumn{1}{|c|}{ Serum TSH } & \multicolumn{1}{c|}{ Serum free T4 } & \multicolumn{1}{c}{ Antithyroid peroxidase } \\
\hline Primary & High & Low & $\begin{array}{l}\text { Positive with Hashimoto/chronic } \\
\text { autoimmune thyroiditis }\end{array}$ \\
\hline $\begin{array}{l}\text { Secondary } \\
\text { TSH, thyroid-stimulating hormone. }\end{array}$ & Low or normal & Low & Negative
\end{tabular}

deficient level of circulating thyroid hormone results in the metabolic syndrome, hypothyroidism. This deficiency may be congenital, but is most often acquired. Acquired hypothyroidism develops as a result of a primary disease of the thyroid gland or secondary to disorders of hypothalamic or pituitary origin. ${ }^{3}$ Primary hypothyroidism accounts for $95 \%$ of acquired hypothyroidism. ${ }^{4}$ Causes include circulating antithyroid antibodies resulting in defective hormone synthesis (autoimmune thyroiditis); damage to or destruction of the thyroid gland during surgery, radiation, or radioactive iodine therapy; antithyroid drugs; and endemic iodine deficiency. ${ }^{2}$ The most common cause of primary hypothyroidism in countries with iodine sufficiency such as the United States is chronic autoimmune thyroiditis (Hashimoto thyroiditis) that results in thyroid-stimulating hormone (TSH) receptor injury and decreased thyroid hormone production. ${ }^{2}$ When thyroid hormone is decreased, the hypothalamus responds with an increase in synthesis of thyrotropin-releasing hormone, which in turn increases secretion of TSH from the anterior pituitary gland. It is the trophic action of increased TSH that may lead to thyroid enlargement or goiter with hypothyroidism. ${ }^{3}$

\section{Clinical presentation}

Hypothyroidism may affect all body functions as reflected in its clinical manifestations. Common presenting symptoms of hypothyroidism include a gradual onset of weakness and fatigue, weight gain, constipation, and cold intolerance. The skin often becomes dry and rough, and hair becomes coarse and brittle. Reproductive-age women may experience menstrual irregularities and infertility. ${ }^{2}$

Myxedematous tissue involvement is mostly in the face. Facial features may be puffy with edematous eyelids and thinning of the outer third of the eyebrows. The tongue may be enlarged and the voice may be hoarse. Thyroid enlargement or goiter may or may not be present. Goiters caused by hypothyroidism are usually nontender and without nodules. Other physical exam findings include hypoactive bowel sounds, delayed relaxation of deep tendon reflexes, and bradycardia. There may be central nervous system involvement with mental dullness, lethargy, and impaired memory. Screening for depression may be warranted. Carpal tunnel syndrome and sleep apnea are associated with severe hypothyroidism. ${ }^{2}$

A transient postpartum thyroiditis occurs in approximately $5 \%$ of women within 12 months postpartum and may include a hyperthyroid phase followed by a hypothyroid phase in which symptoms of fatigue, cold intolerance, dry skin, depression, impaired concentration, and constipation are common. Most cases resolve within one year, but $10 \%$ to $20 \%$ of women remain hypothyroid. $^{5,6}$

\section{Diagnosis}

The diagnosis of hypothyroidism is made based on history, physical exam, and laboratory tests. ${ }^{3}$ Tests to evaluate thyroid function most commonly used in primary care are TSH and serum free thyroxine (FT4). Serum TSH is the most reliable test for hypothyroidism. When primary hypothyroidism is suspected, FT4 and TSH levels should be evaluated. Primary hypothyroidism presents with a high serum TSH concentration and a low serum FT4 concentration. ${ }^{3}$ Tests for antithyroid antibodies may be useful to confirm chronic autoimmune thyroiditis. Antithyroid peroxidase antibodies are commonly detected in patients with Hashimoto thyroiditis. Patients with hypothyroidism secondary to hypothalamic or pituitary gland disorders usually have low or normal TSH levels and low FT4 levels. ${ }^{3}$

See Table for expected lab test results with hypothyroid disorders.

\section{Treatment of primary hypothyroidism in the adult}

Primary hypothyroidism is a chronic condition requiring lifelong therapy of thyroid replacement hormone. Goals of treatment include restoration of the euthyroid state, including normalization of TSH levels, reversal of clinical manifestations of thyroid hormone deficiency, and avoidance of iatrogenic thyrotoxicosis due to overtreatment, especially in the elderly. ${ }^{7}$ Treatment is recommended for all individuals with overt 
primary hypothyroidism and serum TSH greater than $10 \mathrm{mIU} / \mathrm{L}$, regardless of symptoms. ${ }^{2,8}$

\section{Levothyroxine}

Levothyroxine (synthetic thyroxine, T4) is the treatment of choice for hypothyroidism. ${ }^{2,7,8}$ Treatment effectiveness is monitored with standard TSH and FT4 measurements of thyroid function. ${ }^{4}$ Levothyroxine is available in tablet, soft gel capsule, and liquid formulations, with tablets being most commonly prescribed. Soft gel capsules and liquids may improve absorption in patients with atrophic gastritis or following gastric bypass surgery. These two formulations are more costly than tablets, and poor absorption may be successfully addressed by increasing the dose of the tablet form. ${ }^{7}$ Generic and brand-name formulations are equally efficacious. Branded and generic preparations that meet US Food and Drug Administration criteria for potency and bioavailability may be used interchangeably, although the American Academy of Clinical Endocrinologists and the American Thyroid Association recommend assessing thyroid function 4 to 6 weeks after a change in preparation. 2,7

The patient's weight, age, and underlying comorbid conditions should be considered when deciding on the starting dose of levothyroxine. ${ }^{7}$ The dosage required to achieve euthyroidism varies widely, ranging from 50 to $200 \mu \mathrm{g}$ daily. The average full replacement dose of levothyroxine is $1.6 \mu \mathrm{g} / \mathrm{kg} / \mathrm{day}$, with ideal body weight being the best predictor of effective dose. ${ }^{7}$ This is equivalent to 100 to $150 \mu \mathrm{g} /$ day for most young and middle-aged adults and 50 to $75 \mu \mathrm{g} /$ day for most older individuals. Starting with the full replacement dose is acceptable for young and middle-aged healthy patients and will achieve euthyroidism more rapidly than starting at a lower dose and titrating upward. ${ }^{7}$ Patients who are elderly or who have coronary heart disease should be started at a lower dose of 12.5 to $25 \mu \mathrm{g}$ of levothyroxine daily, with gradual adjustments based on serum TSH level and clinical manifestations. ${ }^{7}$

Levothyroxine is best absorbed when taken on an empty stomach, 60 minutes before breakfast or 3 hours after the last meal of the day. ${ }^{7}$ Maintaining a consistent dosing schedule in relation to food intake will achieve acceptable results, as the dose can be adjusted to accommodate the schedule. A patient who has difficulty adhering to the recommended schedule can be counseled on consistency of meals and dosing to achieve desired result. ${ }^{7}$ Medications and supplements that may interfere with absorption or serum concentration should also be separated from the levothyroxine dose. The list of medications that interact with levothyroxine is extensive, and a thorough medication history and interaction check are recommended when initiating and continuing treatment with levothyroxine. Oral (but not transdermal) estrogens increase serum thyroxine-binding globulin levels. Women who are euthyroid compensate with increased thyroid hormone production, but women with a compromised thyroid reserve cannot. Women taking levothyroxine who begin or discontinue oral estrogen therapy may require dosage adjustment based on serum TSH levels. ${ }^{9}$ Vitamin and mineral supplements, including calcium carbonate and ferrous sulfate, and drugs that alter gastric acidity may interfere with absorption of levothyroxine and should be separated from levothyroxine by 4 hours. ${ }^{2,7}$ These supplements are often components of multivitamins, and women taking a multivitamin may also require a higher dose of levothyroxine. $^{7}$

Adverse effects of levothyroxine are rare when dosed appropriately. Allergic reactions to dyes or fillers may occur and should be addressed with a change in formulation. Patients allergic to dyes can be treated with dye-free, white $50-\mu \mathrm{g}$ tablets, with multiple tablets prescribed to achieve the desired dose. Allergies to fillers can be addressed with a change to an alternate formulation, such as soft gel or liquid. ${ }^{7}$ Adverse reactions related to overdosage include cardiovascular manifestations, primarily atrial fibrillation, and accelerated bone loss in postmenopausal women. ${ }^{7}$ Other cardiovascular, central nervous system, endocrine, metabolic, neuromuscular, and gastrointestinal effects may occur as well. ${ }^{10}$ Measures to avoid overtreatment include starting older patients on a lower initial dose, regular monitoring, and patient education. ${ }^{2,7}$ If signs or symptoms of overtreatment occur, TSH should be assessed and the dose adjusted accordingly. ${ }^{7}$

\section{Dietary supplements}

There is a lack of scientific evidence to support the efficacy of dietary supplements or other over-thecounter products for the treatment or prevention of thyroid disease, and their use is not recommended. ${ }^{2,7}$ There is also no evidence to support iodine supplementation beyond the recommended daily allowance (about $150 \mu \mathrm{g}$ ) to enhance thyroid function. ${ }^{2,7}$ lodine supplementation in larger doses can have various effects on thyroid function including the inducement of hyperthyroidism, hypothyroidism, and thyroiditis.

\section{Evaluation and follow-up}

Serum TSH level is the most reliable indicator of thyroid hormone 


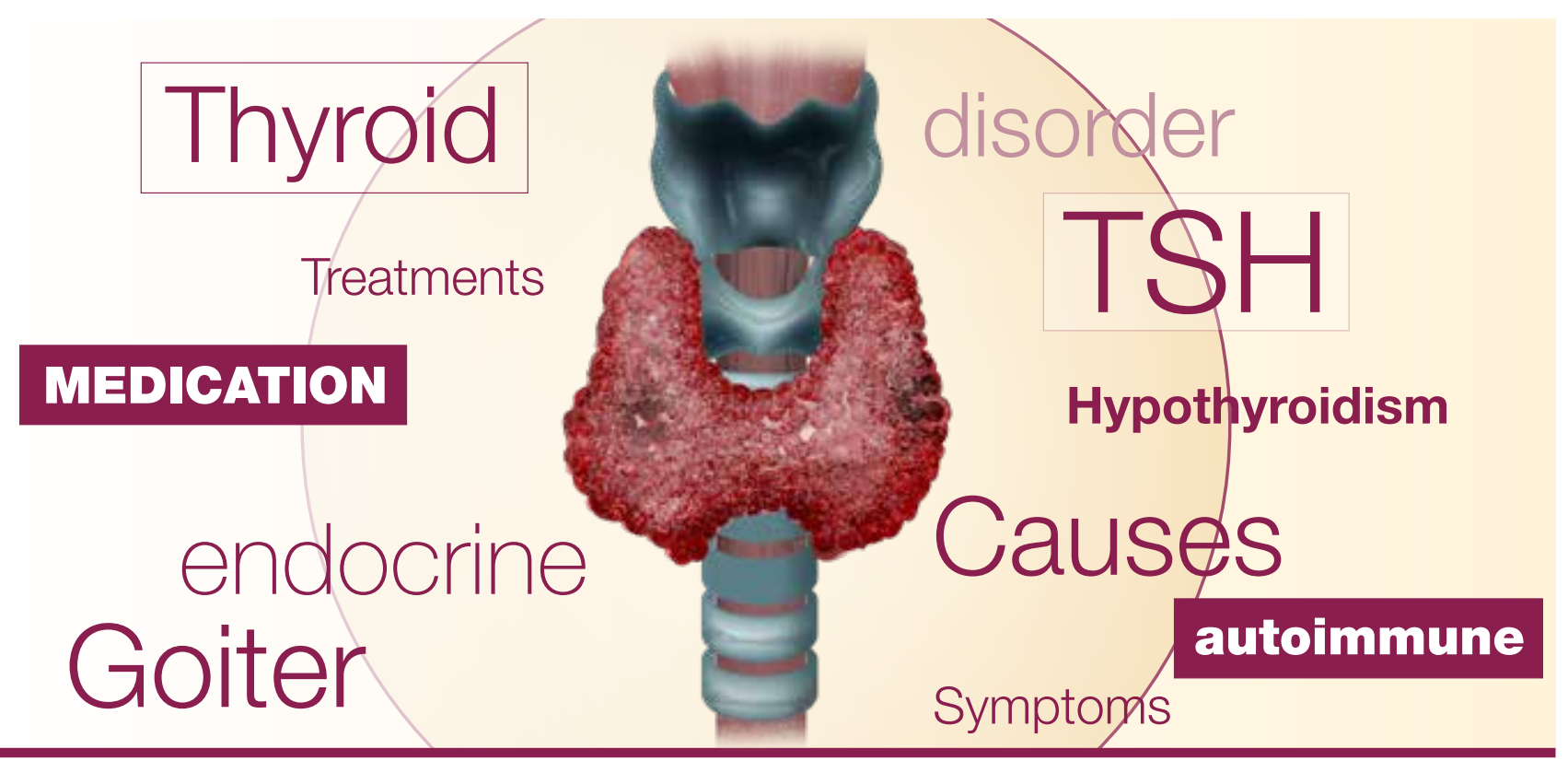

status. ${ }^{2,7}$ Following initiation of thyroid hormone replacement therapy, symptom improvement should be evident within 2 weeks, but complete recovery may take several months. Despite symptom improvement, it will take 6 weeks or longer to reach steady-state TSH levels. ${ }^{7}$ For patients who exhibit symptom improvement, serum TSH should be measured in 4 to 6 weeks. ${ }^{7}$ If serum TSH is above the reference range, the levothyroxine dose should be adjusted in increments of 12.5 to $25 \mu \mathrm{g}$ per day and serum TSH reassessed every 4 to 6 weeks. ${ }^{7}$ If serum TSH is below the reference range, the daily dose should be decreased by 12.5 to $25 \mu \mathrm{g}$ per day and reassessed in 4 to 6 weeks. ${ }^{7}$ If the patient has no symptom improvement after 2 to 3 weeks of thyroid hormone replacement, serum free T4 and TSH should be measured. The dose needs to be assessed and adjusted every 4 to 6 weeks until the euthyroid state is attained. ${ }^{7}$ Once euthyroid status is achieved and the patient's symptoms are stable, TSH and FT4 can be assessed in 4 to 6 months and then yearly. ${ }^{7,8}$ The TSH level should remain between 0.3 and $3.0 \mathrm{mIU} / \mathrm{L}$ during therapy, preferably between
1.0 and $2.0 \mathrm{mIU} / \mathrm{L} .{ }^{7}$ The level of FT4 is more reliable once the patient is stable in the euthyroid state. ${ }^{7}$ It is important to keep FT4 levels stable because elevated levels are associated with sudden cardiac death and accelerated atherosclerosis. ${ }^{4}$

If elevated TSH persists in the presence of high-dose levothyroxine (> $200 \mu \mathrm{g} /$ day), patient adherence to therapy needs to be evaluated. The patient should be assessed and educated about dosing in relation to meals and other medications, and encouraged about consistency in timing of medication and meals. Missed doses should be taken as soon as remembered, even if more than one dose is taken in a single day. If adherence is a significant problem for a patient, the entire weekly dose may be taken in a single day, once a week, with safety and outcomes similar to daily dosing. ${ }^{2}$

If a patient continues to report symptoms of hypothyroidism despite laboratory evidence of euthyroid state, it is important to consider and evaluate the patient for other causes of symptoms. ${ }^{2}$ Increasing the dose of levothyroxine based on symptoms alone increases the risk for overtreatment. ${ }^{7}$ Patients with TSH and FT4 in the normal range, with persistent symptoms, will benefit from referral to an endocrinologist. ${ }^{7}$

Certain conditions may necessitate dosage adjustment after the maintenance dose has been achieved. Conditions that may necessitate increased dosage include pregnancy, weight gain of greater than $10 \%$ of body weight, and conditions that impair thyroid hormone absorption (gastrointestinal conditions) or increase thyroid hormone secretion (nephrotic syndrome), as well as the addition of drugs for coexisting conditions that interfere with thyroid hormone absorption or metabolism. ${ }^{7}$ Conditions that may necessitate a decrease in dosage include normal aging, weight loss of greater than $10 \%$ of body weight, and addition of androgen therapy. ${ }^{7}$

\section{When to consult or refer to an endocrinologist}

Most cases of primary hypothyroidism can be effectively managed by the primary care provider. In certain circumstances, consultation or referral to an endocrinologist is indicated. These circumstances include: difficulty achieving and/or maintaining euthyroid state; infertility; pregnancy 


\title{
The primary goal is to treat with
}

\author{
medications to promote a euthyroid state.
}

$$
\begin{gathered}
\text { Overall, the carefully managed, } \\
\text { euthyroid patient has a good } \\
\text { quality of life. }
\end{gathered}
$$

or planning a pregnancy; cardiac disease; structural changes in the thyroid gland, such as goiter or nodules; comorbid endocrine diseases, including adrenal and pituitary disorders; unusual thyroid function test results; and secondary hypothyroidism. ${ }^{2}$ Appropriate and prompt referral results in more cost-effective care and improved patient outcomes. Individuals with subclinical hypothyroidism $(\mathrm{SCH})$ may also benefit from consultation with an endocrinologist. Subclinical hypothyroidism is diagnosed when TSH is elevated in the presence of normal thyroid hormone levels and the absence of clinical signs and symptoms. Treatment may be indicated for individuals with certain health conditions. ${ }^{2}$

Although $\mathrm{SCH}$ has been associated with infertility and adverse pregnancy outcomes, there is insufficient evidence to recommend treatment for all women with $\mathrm{SCH}$ who are pregnant or hoping to become pregnant. ${ }^{5}$ Women with $\mathrm{SCH}$ who desire pregnancy and are experiencing infertility or have a history of miscarriage should be referred to an endocrinologist or fertility specialist. ${ }^{2}$

The pregnant woman with hypothyroidism will require regular monitoring of TSH levels and adjustment of levothyroxine dosage as needed to maintain a euthyroid state throughout pregnancy. ${ }^{6}$ Women with hypothyroidism typically can return to their prepregnancy levothyroxine dosage after giving birth with a recommended TSH level at 6 weeks postpartum. ${ }^{5,7}$

\section{Conclusion}

The diagnosis of hypothyroidism has a lifelong impact and involves treatment to correct a hypometabolic state. The primary goal is to treat with medications to promote a euthyroid state. Pharmacologic measures are essential for treating the disease, and follow-up is warranted. Overall, the carefully managed, euthyroid patient has a good quality of life. The NP providing healthcare for women can be alert to symptoms of and risks for hypothyroidism including menstrual irregularities, infertility, and postpartum state, as well as other common signs and symptoms. With appropriate diagnostic testing, the NP can initiate treatment with levothyroxine and monitor patient response. Regular follow-up will guide treatment changes and referral to an endocrinologist if necessary.

\section{Cynthia S. Watson is an Assistant Professor and Janis Guilbeau is an Associate Professor at the Univer- sity of Louisiana at Lafayette Col- lege of Nursing. The authors have no actual or potential conflicts of interest in relation to the contents of this article.}

\section{References}

1. Singh S, Clutter WE. Hypothyroidism. In: Baranski TJ, McGill JB, Silverstein JM, eds. Endocrinology Subspecialty Consult, 4th ed. Philadelphia, PA: Wolters Kluwer; 2020:70-76.

2. Garber JR, Cobin RH, Gharib H, et al; American Association of Clinical Endocrinologists; American
Thyroid Association Taskforce on Hypothyroidism in Adults. Clinical practice guidelines for hypothyroidism in adults: cosponsored by the American Association of Clinical Endocrinologists and the American Thyroid Association. Endocr Pract. 2012;18(6):988-1028.

3. Norris TL, Lalchandani R. Porth's Pathophysiology: Concepts of Altered Health States. 10th ed. Philadelphia, PA: Wolters Kluwer; 2019.

4. Woo TM, Robinson MV. Pharmacotherapeutics for Advanced Practice Nurse Prescribers. 5th ed. Philadelphia, PA: FA Davis Company; 2020.

5. Alexander EK, Pearce EN, Brent GA, et al. 2017 Guidelines of the American Thyroid Association for the diagnosis and management of thyroid disease during pregnancy and the postpartum. Thyroid. 2017;27(3):315-389.

6. American College of Obstetricians and Gynecologists. Practice Bulletin No. 223: Thyroid disease in pregnancy. Obstet Gynecol. 2020;135(6):e261-274.

7. Jonklaas J, Bianco AC, Bauer AJ, et al. Guidelines for the treatment of hypothyroidism: prepared by the American Thyroid Association Task Force on Thyroid Hormone Replacement. Thyroid. 2014;24(12):1670-1751

8. Chaker L, Bianco AC, Jonklaas J, Peters R. Hypothyroidism. Lancet. 2017;390(10101):1550-1562.

9. The North American Menopause Society. Menopause Practice: A Clinician's Guide. 6th ed. Pepper Pike, OH: The North American Menopause Society; 2019.

10. Lexicomp. Levothyroxine: drug information. May 28, 2020. https:// go.wolterskluwer.com/LCO. 